\title{
Septic Arthritis in Children: A Longitudinal Population-Based Study in Western Australia
}

\author{
Johannes C. Nossent (D) - Warren D. Raymond • Helen I. Keen • \\ Charles A. Inderjeeth
}

Received: March 5, 2021 / Accepted: April 9, 2021 / Published online: April 25, 2021

(C) The Author(s) 2021

\section{ABSTRACT}

Objective: To describe the incidence, risk factors and long-term outcomes in children hospitalised with septic arthritis (SA) in Western Australia (WA).

Methods: We extracted state-wide longitudinally linked administrative health data for patients aged $<16$ years with a first diagnostic code of 711.X (ICD9-CM) and M00.X (ICD10AM) in WA in the period 1990-2010. Annual incidence rates (AIR) per 100,000 with 95\% confidence intervals (CIs), prior conditions during a median lookback period of 63.2 [interquartile range (IQR) 19.8-117.1] months and outcomes, including standardised mortality

Supplementary Information The online version contains supplementary material available at https:// doi.org/10.1007/s40744-021-00307-x.

J. C. Nossent · C. A. Inderjeeth

Department of Rheumatology, Sir Charles Gairdner Hospital, Perth, Australia

J. C. Nossent $(\bowtie)$. W. D. Raymond .

H. I. Keen - C. A. Inderjeeth

Rheumatology Group, School of Medicine,

University of Western Australia, 35 Stirling Highway

(M503), Perth, WA, Australia

e-mail: johannes.nossent@uwa.edu.au

H. I. Keen

Department of Rheumatology, Fiona Stanley

Hospital, Perth, Australia rates (SMR), during a median follow-up of 10 years are reported.

Results: A total of 891 patients [62\% male, median age 6.4 (IQR 1.9-10.6) years with $34 \%$ aged $<3$ years] were admitted for SA during the observation period. The overall AIR (per $100,000)$ was 9.85 (95\% CI 4.79-14.41), and was higher in Indigenous Australians [34.9 vs. 5.5 (non-Indigenous), $p<0.001]$ and in males [11.9 vs. 7 (females), $p<0.01$ ]; AIR showed no temporal or seasonal variation. Knees (43.9\%), hips (34.6\%) and ankles (13.3\%) were most frequently affected, with Staphylococci predominant $(49 \%)$ in patients with positive cultures (41.5\%). Prior infection(s) (40.4\%) and respiratory disease $(7 \%)$ were the main pre-existing morbidities. Median hospital stay was 4.0 (IQR $2-8$ ) days, with $1.9 \%$ requiring admission to the intensive care unit and $10.4 \%$ requiring readmission within 30 days. During follow-up, 26 patients (3.1\%) developed osteomyelitis, nine patients were diagnosed with osteoarthrosis $(1.1 \%)$ and five patients $(0.6 \%)$ underwent joint replacement. Female patients developed other serious infections more often than male patients (40.5 vs. $27.1 \%, p<0.01)$, as well as other comorbidities (Charlson Comorbidity Index $>0: 34.6$ vs. $27.2 \%, p=0.02$ ), including diabetes ( 4.2 vs. $0 \% ; p=0.001)$, cardiovascular events ( 4.2 vs $1.4 \%, p=0.002$ ) and chronic arthritis ( 1 vs. $0 \%, p=0.05$ ). The crude mortality rate was low $(0.3 \%)$, with $99.4 \%$ survival at 180 months and no increase in the SMR. 
Conclusions: The incidence of SA in children in WA did not change over the 20-year observation period. SA did not lead to excess mortality, but bone and joint complications developed in $5 \%$ of patients. The high propensity to comorbid conditions in this young cohort suggests an underlying role of comorbidity in SA development.

\section{PLAIN LANGUAGE SUMMARY}

As more children are living with complex and chronic conditions, we investigated whether children in Western Australia (WA) have become more prone to joint infections. During a 20-year observation period we collected health data for all children admitted to any hospital in the state with an infected joint and recorded their health outcomes. We found that joint infection occurs in nearly ten out of 100,000 children each year, but we saw no change in the frequency over time. We did observe higher rates in Indigenous children $(35 / 100,000)$ than in non-indigenous children $(6 / 100,000)$ but found no noticeable influence of the seasons on the frequency of joint infections. Knees, hips and ankles were most often affected, and 15\% had additional bone infection. Children needed to be treated in hospital for 4-5 days, and only a small minority $(1.2 \%)$ were so ill they needed intensive care. Joint infections led to chronic, long-term complications in about 5\% of patients, but we found no evidence that joint infections increased the risk of death compared to children in the general population.

Keywords: Septic arthritis; Paediatric; Incidence; Morbidity; Mortality

\section{Key Summary Points}

The overall incidence of septic arthritis (SA) in children (10/100,000/year) in Western Australia did not change over a 20 -year period and showed no seasonal variation.

Indigenous Australian children had a sixfold higher incidence rate of SA than non-Indigenous children.

There was no excess risk of death following SA, but $5 \%$ of children required subsequent hospitalisation for bone/joint abnormalities.

A high frequency of prior hospitalisation and subsequent comorbidity development, including other infections, suggest a role of comorbidity in predisposing children to SA.

\section{DIGITAL FEATURES}

This article is published with digital features, including a summary slide and plain language summary to facilitate understanding of the article. To view digital features for this article go to https://doi.org/10.6084/m9.figshare. 14387522

\section{INTRODUCTION}

Septic arthritis (SA) develops from the invasion of microorganisms into the lining of synovial joints $[1,2]$. It is most frequently the result of hematogenous spread of microorganisms to the synovial membrane, although bacteria can also be introduced directly into the joints by trauma or spread from nearby inflamed tissues. The probability to develop SA following bacteraemia is between 12 and $17 \%$ depending on a variety of microorganism and host factors [3, 4]. Once inside the synovium, bacteria will proliferate and trigger inflammatory cellular (neutrophils 
an monocytes) and humoral (complement, cytokines) responses that contribute to the inflammatory destruction of joint structures in SA [3]. SA is considered to be a medical emergency as it can lead to the rapid destruction of joints with the potential for poor functional outcomes [5-9]. Reports on the incidence of SA in children are sparse, with studies from the USA reporting an annual incidence rate (AIR) of 4 per 100,000 person-years, while a recent study from Israel reported an AIR of 11.7 per 100,000 person-years $[10,11]$. SA incidence in adults has been rising over the last 25 years due to a combination of multi-comorbidity, iatrogenic complications and increasing use of immunomodulating therapies [12-15]. It is conceivable that this scenario applies to children as well, although data from the USA suggest no major changes over time [9]. As AsianPacific data on SA are sparse and limited to case series [16-19], the aim of this study was to investigate the state-wide frequency, underlying clinical characteristics and outcomes of incident SA in children in Western Australia (WA) over the period 1990-2010.

\section{METHODS}

This was a population-based retrospective cohort study that used routinely collected statewide administrative health data for patients with specific rheumatic diseases recorded in the Western Australian Rheumatic Disease Epidemiological Registry between 1980 and 2015 (WARDER) [20, 21]. WARDER data were extracted and linked through the Western Australian Data Linkage System (WADLS) using probabilistic matching (based on a person's name, residential address, date of birth and gender) to link all health contacts over time for each individual registered in the Hospital Morbidity Data Collection (HMDC), WA Cancer Registry, WA Mortality Registry or the Emergency Department Data Collection (EDDC) in WA (population 2.5 million). More detailed information on the data linkage process is readily available online (https://www. datalinkage-wa.org.au). The high linkage accuracy $(99.7 \%)$ and compulsory reporting of data allow longitudinal follow-up of individuals across administrative health datasets. For this study we used HMDC and EDDC data from all public and private hospitals in WA.

\section{Study Cohort}

Patients with incident SA were identified by at least one International Classification of Diseases (ICD) code for pyogenic arthritis (ICD-9-CM: 711.xx; ICD-10-AM: M00.xx) in the HMDC and EDDC between 1 January 1990 and 31 December 2010 to ensure a minimum follow-up of 5 years. Notably, we did not include patients with infectious arthritis occuring in conjunction with infectious and parasitic diseases classified elsewhere, such as gonococcal (ICD-10-AM: M54) and tuberculous (M01.1), Lyme disease (M01.2), viral (M01.4-5), mycotic (M01.6), and parasitic (M01.8) joint infections. We subsequently excluded all patients aged $>16$ years at diagnosis and all patients with a diagnostic code for prosthetic joint infection (ICD-9-CM: 996.60-996.67; ICD-10-AM: T84.5-84.7, Y83, Y83.8 and E878.1). We defined the first date with a SA diagnostic code as time zero $\left(T_{0}\right)$. As $T_{0}$ was not necessarily the first hospital contact, we defined a lookback period as all observation time prior to $T_{0}$ and a follow-up time as all observation time after $T_{0}$ to date of death or exit date of 31 December 2014. We recorded specific as well as global comorbidity according to the validated and prognostically important Charlson comorbidity index (CCI) $[22,23]$. We also registered the occurrence of other serious infections during follow-up across all hospital contacts defined as episodes leading to Emergency Department (ED) presentation and/or hospital admission resulting in a validated infectious disease code for pneumonia, sepsis or bacteraemia, urinary tract infection and skin and soft tissue infections [24]. Primary outcomes were SA incidence rate per 100,000 population per annum; the presence of pre-existing comorbidity as risk factors for SA; SA recurrence rate; and, post-SA bone and joint complications as well as surgery rates. Relevant diagnostic codes are provided in Electronic Supplementary Material (ESM) Table 1. All-cause mortality per 
A 16.00

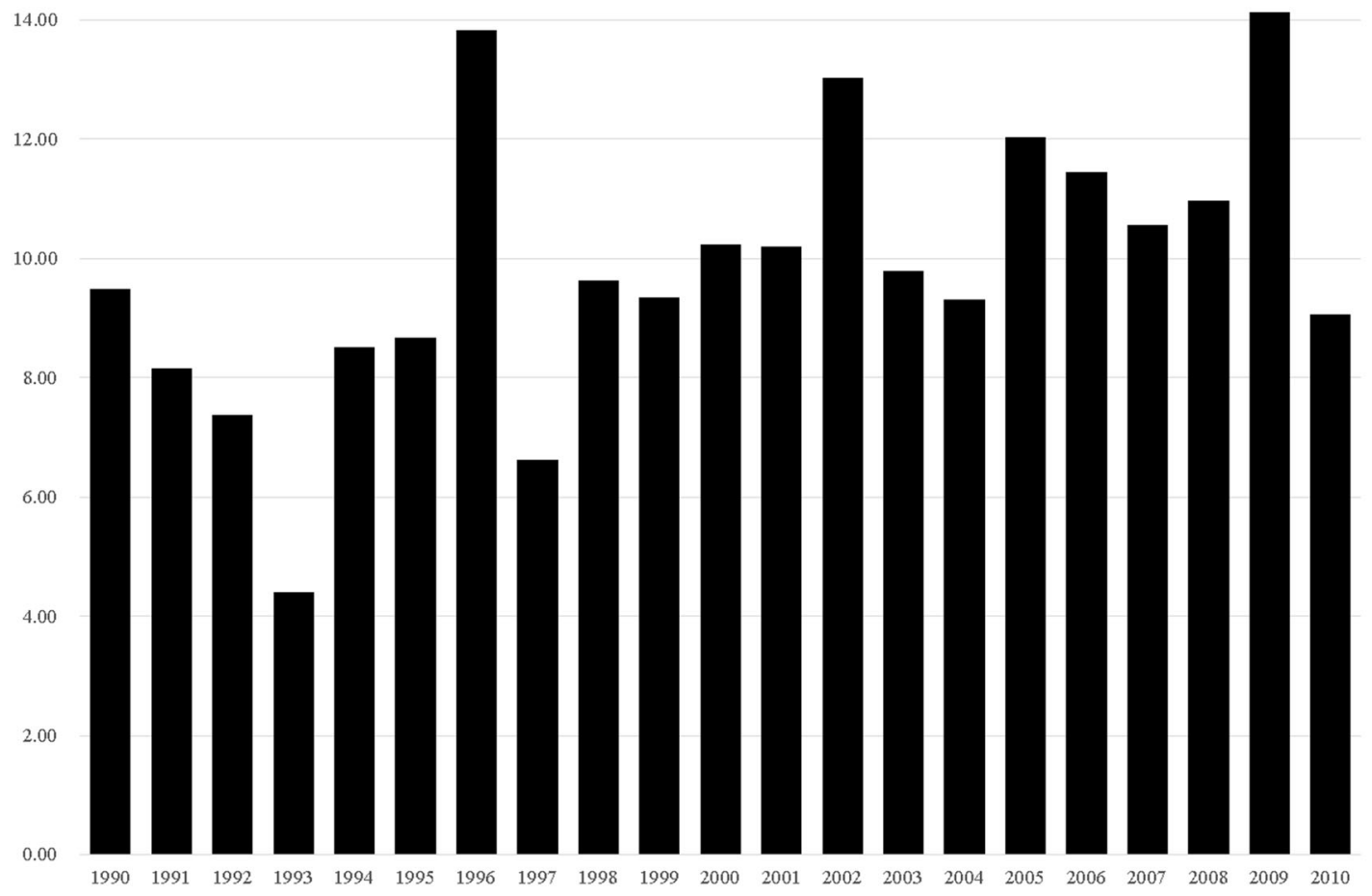

B

-non-IA - -IA
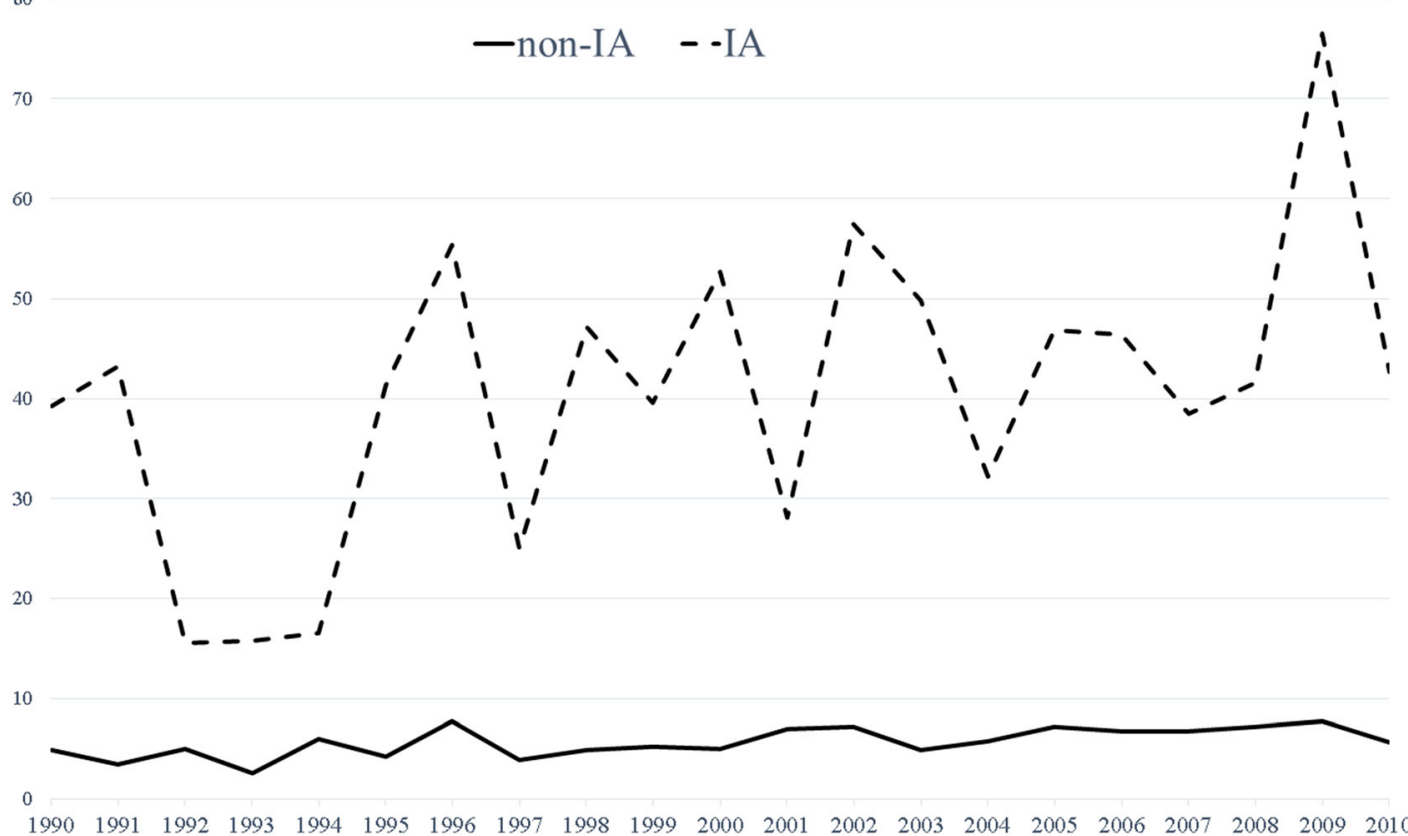
4Fig. 1 Overall annual incidence of septic arthritis per 100,000 population aged < 16 years (a) in Western Australia over period 1990-2010 and annual incidence by ethnic background (b). IA Indigenous Australians

1000 person-years [mortality rate (MR)] and survival estimates were based on date of death in the WA Death Registry. Approval for use of the de-identified dataset was obtained from the Human Research Ethics Committee at the WA Department of Health (WADOH HREC\# 2016.24).

\section{Statistical Analyses}

Descriptive statistics include the median and interquartile range (IQR) and mean with standard deviation for continuous variables, compared by non-parametric methods (Kruskal-Wallis). Categorical data are described with a frequency and proportion. Group comparisons are tested with the Fisher's exact test. Incidence rates for SA are given per 100,000 population for each study year, and the standardised MR (SMR) with Poisson-derived 95\% confidence intervals (95\% CIs) are based on ageand gender-specific MR obtained from the Australian Bureau of Statistics (https://www.abs. gov.au/statistics/people/population/nationalstate-and-territory-population/latestrelease\#data-download). All statistics were performed using SPSS software v23.0 (IBM Corp., Armonk, NY, USA) and OpenEpi software with only two-sided $p$ values. $p<0.05$ was considered to be statistically significant.

\section{RESULTS}

A total of 891 children [61.9\% males; median age 6.3 (IQR 1.9-10.7) years] were diagnosed with SA over the 20-year observation period. The overall AIR was 9.85 (95\% CI 4.79-14.41) and did not change notably over time (Fig. 1a). Most patients $(71.9 \%)$ resided in the metropolitan area, but there was a high proportion of patients of Indigenous background (Table 1) in whom overall AIR was 34.9 (95\% CI 25.3-46.4) versus 5.6 (95\% CI 2.8-10.2) in non-
Indigenous patients (Fig. 1b). The predominance of males was sustained over time (ESM Fig. 1), but there was no seasonal influence on SA presentation (ESM Fig. 2). Other than a higher proportion of female patients aged $<3$ years, there were few gender-specific differences in disease presentation (Table 1). The predominant infective organism in patients was Staphylococcus (49\%), occurring in $41.5 \%$ of positive cultures, with the knees (43.9\%), hips (34.6\%) and ankles (13.3\%) the most frequently infected. Concurrent osteomyelitis occurred in $16.3 \%$ of patients. Median hospital stay was 4.0 (IQR 2-8) days, and nearly $2 \%$ of patients were admitted to the Intensive Care Unit, while readmission rate within 31 days and in-hospital MR were 10.4 and $0.24 \%$, respectively (Table 1 ).

SA was the reason for the first ever hospital contact for 211 patients $(24.7 \%)$, while the remaining 680 patients had 3901 earlier hospital visits (median 5, IQR 2-12) during a median lookback period of 63.2 (IQR 19.8-117.1) months. Clinically diagnosed (52.9\%) and culture-confirmed infections $(38.2 \%)$ were the most frequent pre-existing conditions (Table 1), while chronic conditions as defined by the CCI were present in $116(17.1 \%)$ patients, with most due to chronic respiratory disease $(9.3 \%)$ and liver disease (7.6\%); immunodeficiency $(0.4 \%)$ and rheumatic disease $(0.3 \%)$ were relatively rare.

Follow-up data beyond 30 days were available for 832 patients (93.4\%) with a median age at last observation of 18.7 (IQR 11.9-24.2) years and across 3099 hospital admissions and 7404 ED visits during a median observation period of 131 (IQR 74-207) months. Twelve patients (1.4\%) had a second admission for SA at median period of 4 (IQR 2-6) months after the initial admission for SA, and 26 patients (3.1\%) were subsequently admitted for osteomyelitis after a median of 7 (IQR 2-30.5) months (Table 2). Only one patient (3.8\%) with late-onset osteomyelitis had experienced a recurrence of SA. Osteoarthrosis was diagnosed in nine patients after a median follow-up of 122 (IQR 54-218) months, resulting in two joint replacement surgeries. Ankylosis, contractures and dislocations were rare as a cause for readmission (0.2\%). Liver disease was the 
Table 1 Demographic and clinical data at time of diagnosis of septic arthritis

\begin{tabular}{|c|c|c|c|c|}
\hline Demographic and clinical data at diagnosis ${ }^{a}$ & $\begin{array}{l}\text { Overall }(N= \\
891)\end{array}$ & $\begin{array}{l}\text { Male } \\
(n=552)\end{array}$ & $\begin{array}{l}\text { Female } \\
(n=339)\end{array}$ & $p$ value \\
\hline Median age (IQR) & $6.3(1.9-10.7)$ & $6.7(1.1-11.1)$ & $4.9(1.0-10.1)$ & $<0.01$ \\
\hline Age $<3$ years & $305(34.2)$ & $171(31)$ & $134(39.5)$ & 0.006 \\
\hline Metropolitan area & $634(71.2)$ & $390(70.6)$ & $244(71.9)$ & 0.7 \\
\hline Rural area & $257(28.8)$ & $162(29.4)$ & $95(28.1)$ & - \\
\hline Indigenous background (\%) & $277(31.1)$ & $168(30.4)$ & $109(32)$ & 0.72 \\
\hline \multicolumn{5}{|l|}{ Affected joints ${ }^{b}$} \\
\hline Knee & $392(43.9)$ & $267(39.1)$ & $125(36.9)$ & 0.38 \\
\hline Hip & $309(34.6)$ & $180(32.6)$ & $129(38)$ & 0.17 \\
\hline Ankle & $110(12.3)$ & $67(12.2)$ & $43(12.7)$ & 0.31 \\
\hline Elbow & $49(5.5)$ & $35(6.3)$ & $14(4.2)$ & 0.21 \\
\hline Shoulder & $38(4.3)$ & $22(4.0)$ & $16(4.7)$ & 0.83 \\
\hline Wrist & $28(3.2)$ & $17(3.1)$ & $11(3.3)$ & 0.9 \\
\hline Hand & $27(3.0)$ & $15(2.7)$ & $12(3.5)$ & 0.64 \\
\hline Concurrent osteomyelitis & $145(16.3)$ & $88(15.9)$ & $57(16.8)$ & 0.73 \\
\hline Joint procedure performed & $539(60.5)$ & $325(58.8)$ & $214(63.1)$ & 0.26 \\
\hline Concurrent other infections $s^{c}$ & $98(11)$ & $63(11.4)$ & $35(10.3)$ & 0.56 \\
\hline $\begin{array}{l}\text { Microrganism identified (\% of all microorganisms } \\
\text { identified) }\end{array}$ & $370(41.5)$ & $224(40.5)$ & $146(43.1)$ & 0.42 \\
\hline Staphylococcus & $180(48.6)$ & $116(51.7)$ & $64(43.8)$ & \\
\hline Streptococcus & $52(14.1)$ & $32(14.3)$ & $20(7.3)$ & \\
\hline Other & $138(37.3)$ & $76(33.9)$ & $62(42.4)$ & \\
\hline Patients with earlier hospital contacts & $680(76.3)$ & 438 & 242 & \\
\hline Preexisting morbidity $(\mathrm{CCI}>0)$ & $116(13)$ & $85(19.4)$ & $31(12.8)$ & 0.033 \\
\hline Chronic respiratory disease & $63(7.1)$ & $50(11.4)$ & $13(5.4)$ & 0.009 \\
\hline Liver disease & $52(5.8)$ & $37(8.4)$ & $15(6.2)$ & 0.36 \\
\hline Number with any previous infection & $360(40.4)$ & $234(53.4)$ & $126(52.1)$ & 0.68 \\
\hline Number with $>1$ infectious episode & $211(23.7)$ & $143(32.6)$ & $68(28.1)$ & 0.05 \\
\hline Median length of stay, days (IQR) & $4(2-8)$ & $4(1-7)$ & $5(2-8)$ & 0.52 \\
\hline Admitted to ICU & $17(1.9)$ & $9(1.6)$ & $8(2.3)$ & 0.47 \\
\hline
\end{tabular}


Table 1 continued

\begin{tabular}{|c|c|c|c|c|}
\hline Demographic and clinical data at diagnosis ${ }^{a}$ & $\begin{array}{l}\text { Overall }(N= \\
891)\end{array}$ & $\begin{array}{l}\text { Male } \\
(n=552)\end{array}$ & $\begin{array}{l}\text { Female } \\
(n=339)\end{array}$ & $p$ value \\
\hline Patients readmitted within 31 days & $93(10.4)$ & $53(10.2)$ & $40(11.8)$ & 0.48 \\
\hline
\end{tabular}

commonest comorbidity accrued during the follow-up of male and female patients (24.9 vs. $22.2 \%, p=0.390)$. Female patients accrued more comorbidity than male patients (34.6 vs. $27.2 \%, p=0.028$ ) during follow-up, with a higher proportion of hospitalisation for diabetes mellitus, infection and cardiovascular disease. This was associated with events. Longterm complications were as frequent in patients with culture-positive SA at baseline as in those with culture-negative $\mathrm{SA}$ at baseline (data not shown, all $p>0.1$ ), including a non-significant $13 \%$ higher risk of accruing comorbidity $(\mathrm{CCI}>0)$ for culture-positive patients (odds ratio [OR] 1.13, 95\% CI 0.00-1.29, $p=0.08$ ). Indigenous patients were at higher odds for developing osteomyelitis (OR 2.42, 95\% CI 1.14-5.18, $p=0.02$ ) and attaining a CCI $>0$ (OR 1.29, 95\% CI 1.14-1.46, $p<0.01$ ). Following SA, there was a significant use of healthcare resources, such as ED visits and subsequent hospital admission, which was more pronounced in female patients (Table 2). Overall survival at 180 months of follow-up was $99.4 \%$, with a MR in male patients of 0.17 (95\% CI $0.09-0.88)$ and in females of 0.28 (95\% CI 0.04-1.58); both values are not different from those expected by population data (Table 2).

\section{DISCUSSION}

We found that the incidence of native joint SA in children did not change in WA between 1990 and 2010, was highest in Indigenous children and that while SA resulted in subsequent hospitalisation for bone and joint complications in
$5 \%$ of patients, SA did not increase the risk of death.

The AIR of paediatric SA in WA falls within the reported incidence range from 4 to 12 per $100,000[10,25-27]$. While a study from Norway reported an AIR of 5 per 100,000 based on 12 cases over a 1-year period, a recent long-term study based on the Kids' Inpatients Database (KID) in the USA reported a stable AIR of 5.5 over two decades, which is in agreement with the AIR of 5.6 in non-Indigenous patients in WA. In contrast, the high AIR in Indigenous children (which make up approximately $6 \%$ of the total population aged $<16$ years) support findings by Okuzo et al. [11] and recent data from Israel [10] which demonstrated significantly higher rates in disadvantaged children and illustrate the importance of ethnicity and socioeconomic status in the incidence and hospitalization rates of SA. As in other studies, there was no large change in SA incidence over time $[10,26]$, suggesting a limited effect of changes in medical practice, such as the increased use of immune suppression and invasive joint procedures for children [12]. While the incidence of SA was higher in winter and autumn in Norway [28], we found no seasonal effect on SA incidence in the Mediterranean and tropical climate of WA.

The higher proportion of male patients and a predominance of large and lower extremity joints being affected fits well with the literature $[11,26,28]$, although reasons for the gender discrepancy remain unexplained, especially given the similar rates of pre-existing infections (Table 1). Concurrent osteomyelitis was diagnosed in $16 \%$ of all SA patients, and although at 
Table 2 New complications in patients following septic arthritis in childhood

\begin{tabular}{|c|c|c|c|}
\hline New complications & Males $(n=520)$ & Females $(n=309)$ & $p$ value \\
\hline Median follow-up (months) & 134 (IQR 73-198) & 127 (IQR 74-215) & 0.66 \\
\hline Median age last observation (years) & 18.7 (IQR 12.9-23.8) & $18.6(\mathrm{IQR} 10.8-24.5)$ & 0.56 \\
\hline Number of patients with recurrent $S A>30$ days & $6(1.2)$ & $6(1.9)$ & 0.37 \\
\hline Months since first SA event & $4(\mathrm{IQR} 2-4.5)$ & $4(\mathrm{IQR} 3.5-6)$ & 0.36 \\
\hline Number of patients with osteomyelitis & $13(2.7)$ & $13(4.5)$ & 0.16 \\
\hline Months since SA event & 7 (IQR 2-21) & 7 (IQR 2-45) & 0.65 \\
\hline Number of patients with osteoarthrosis & $<5(0.3)$ & $7(2.3)$ & 0.02 \\
\hline Months since SA event & 88 (IQR 54-88) & 143 (IQR 85-218) & 0.88 \\
\hline Number of patients with subsequent joint aspiration & $16(3.1)$ & $18(5.8)$ & 0.07 \\
\hline Months since SA event & $54(\mathrm{IQR} 7-135)$ & 15 (IQR 3-47) & 0.12 \\
\hline Number of patients with joint replacement surgery & $<5(0.4)$ & $<5(1)$ & 0.14 \\
\hline Number of patients with peripheral fracture & $8(1.5)$ & $<5(0.3)$ & 0.17 \\
\hline Number of patients with any comorbidity $(\mathrm{CCI}>0)$ & $142(27.2)$ & $107(34.6)$ & 0.028 \\
\hline Number of patients with cardiovascular events & $6(1.2)$ & $13(4.2)$ & 0.002 \\
\hline Number of patients with congestive heart failure & $<5(0.2)^{\mathrm{a}}$ & $<5(1.9)$ & 0.012 \\
\hline Number of patients with venous thrombosis & $<5(0.4)$ & $<5(1)$ & 0.36 \\
\hline Number of patients with stroke & $<5(0.4)$ & $<5(1.3)$ & 0.21 \\
\hline Number of patients with liver disease & $116(22.2)$ & $77(24.9)$ & 0.39 \\
\hline Number of patients with diabetes mellitus & - & $13(4.2)$ & 0.001 \\
\hline Number of patients with Immune arthritis arthropathy & - & $<5(1)$ & 0.05 \\
\hline Number of patients with renal disease & $5(1)$ & $<5(1.3)$ & 0.73 \\
\hline Number of patients with malignancy & $<5(0.6)$ & $6(1.9)$ & 0.24 \\
\hline Number of patients with immune deficiency & $<5(0.8)$ & $<5(0.6)$ & 0.91 \\
\hline Number of patients with pneumonia & $28(5.4)$ & $26(8.4)$ & 0.11 \\
\hline Number of patients with sepsis & $6(1.2)$ & $<5(1.3)$ & 0.9 \\
\hline Number of patients with urinary tract infection & $6(1.2)$ & $38(12.3)$ & 0.001 \\
\hline Number of patients with skin/soft tissue infection & $80(15.2)$ & $47(15.4)$ & 0.9 \\
\hline Number of patients with (culture-positive) infection & $141(27.1)$ & $125(40.5)$ & 0.001 \\
\hline Number of patients with Staphylococcus infection & $43(8.3)$ & $38(12.3)$ & 0.07 \\
\hline Median number of Emergency Department visits & 12 (IQR 7-24) & 17 (IQR 8-39) & 0.001 \\
\hline Median number of admissions & 3 (IQR 1-8) & 8 (IQR 4-16) & 0.001 \\
\hline Mortality rate/1000 person-years & $0.18(95 \%$ CI $0.002-1.0)$ & 0.28 (95\% CI 0.04-1.58) & 0.76 \\
\hline
\end{tabular}


Table 2 continued

\begin{tabular}{lccc}
\hline New complications & Males $(\boldsymbol{n}=\mathbf{5 2 0})$ & Females $(\boldsymbol{n}=\mathbf{3 0 9})$ & $\boldsymbol{p}$ value \\
\hline Standardised mortality rate & $0.72(95 \%$ CI $0.40-3.67)$ & $1.55(95 \%$ CI 0.08-7.81) & - \\
\hline $\begin{array}{l}\text { Values in table are presented as median values with the IQR in parentheses or as frequency with the percentage in } \\
\text { parentheses, unless indicated otherwise }\end{array}$ & \\
$S A$ Septic arthritis \\
${ }^{\text {a }}$ Small numbers $(n<5)$ have been confidentialised due to HREC requirements
\end{tabular}

the lower end of reported rates, it clearly illustrates that SA and osteomyelitis can occur simultaneously. Assessment for osteomyelitis thus remains an important part of the evaluation of a child with SA and vice-versa, as $20-40 \%$ will experience an infection of the adjacent joint $[29,30]$.

The high prevalence of staphylococcal infections in paediatric SA is well recognised and our data confirm this trend. The overall rate of positive cultures in this study was $41.5 \%$, which is in line with other studies that do not solely rely on the presence of a positive culture to diagnose SA [31]. Given the fact that cultures often remain negative [32-35], this should also not deter physicians from instituting therapy for presumed SA in a compatible clinical picture as this may lead to poor outcomes [1]. While an increasing role for Kingella infections has been described in culture-negative paediatric SA $[31,36]$, we were unable to investigate the role of this relatively new agent as there is no Kingella-specific diagnostic code in discharge summaries.

The average length of hospital stay (LOS) (5.8 days) in our study is in agreement with US data [26], but is shorter than the reported 9.2 days in the Israeli study [10]. The rather short LOS is somewhat surprising but in keeping with recommendations that short intravenous antibiotic regimens followed by oral therapy are not inferior to long term intravenous therapy with regards to long-term outcomes [37, 38]. However, the $11 \%$ readmittance rate in the first month following discharge suggests that this may not hold true in the shorter term for all patients.

The most positive finding regarding longterm outcome is that SA in childhood did no impact on mortality outcome over a 10-year follow-up period, which agrees with US data [36] but contrasts with a recent Nigerian study reporting a 2.3\% short-term MR related to delayed presentation [37] and a 7.3\% MR reported from Turkey [38]. No long-term bone and joint complications were observed in 95\% of all patients, confirming that most children will recover from SA without major functional problems [36].

However, as our data did not capture outpatient or allied health visits, minor disabilities cannot be fully excluded [15]. The $3.1 \%$ frequency of late-onset osteomyelitis is intriguing since it is less likely (but not impossible) given the time elapsed that this reflects smouldering osteomyelitis already present at SA. Otherwise, it seems to suggest an increased propensity to new osteoarticular infections in a subgroup of patients for reasons that are unclear. Diabetes mellitus and immune arthritis could be contributing factors but do not fully explain this as they solely occurred in female patients. The high frequency of SA patients with (mostly mild) liver disease is likely a result of the overrepresentation of young adult Indigenous patients, who have sixfold higher rates of liver disease. There was a significant use of healthcare resources in this cohort, with infectious disease as the main cause of subsequent hospital visits. Together with the high rate of infectious disease observed before SA diagnosis (Table 1), this indicates that SA patients overall have a higher propensity to severe infections, but data on other potential immune deficiencies underlying SA are rare.

The limitations of this study deserve mentioning. Our AIR data are minimum estimates as we limited the diagnostic classification of SA to 
the most common subtype of pyogenic arthritis. Inclusion of other types of bacterial arthritis, as in some other studies, would thus lead to a further increase in AIR. We did not exclude patients with inconclusive cultures, which complicates comparison with culture-positive case series, but our results suggest that cultures did not have a significant impact on long-term outcomes. Finally, the nature of our administrative database did not allow astudy of clinical details, such laboratory findings, type and duration of antibiotic or other drug therapy or microbiological susceptibility. On the other hand, the large number of patients and the comprehensive state-wide data over a lookback period as well as long-term follow-up with validated endpoints strengthen the results.

\section{CONCLUSION}

Septic arthritis has not become more frequent over a 20-year period. It is not seasonal, but continues to affect Indigenous children in WA disproportionally. Although SA does not increase the risk of death, it leads to long-term bone/joint complications in 5\% of patients.

\section{ACKNOWLEDGEMENTS}

The authors would like to acknowledge the support of the Arthritis Foundation of WA and acknowledge the Western Australian Data Linkage Branch, the Western Australian Department of Health and the data custodians of the Hospital and Morbidity Data Collection, the Emergency Department Data Collection, the WA Cancer Register and the WA Death Register for their assistance with the study. This work was supported by an unrestricted grant from the Arthritis Foundation of Western Australia to JCN. Author WDR is the recipient of the John Donald Stewart Scholarship from the Arthritis Foundation of Western Australia. No support was received for medical writing or editorial assistance
Funding. No funding was received for the publication of the article.

Authorship. All named authors meet the International Committee of Medical Journal Editors (ICMJE) criteria for authorship for this article, take responsibility for the integrity of the work as a whole, and have given their approval for this version to be published.

Authors' Contributions. Study design: JCN, WDR, HIK and CAI. Data extraction and analysis: JCN, WDR. Drafting/critical reading: JCN, WDR, HIK and CAI.

Disclosures. Johannes C Nossent, Warren D Raymond, Helen I Keen and Charles A Inderjeeth have nothing to disclose.

Compliance with Ethics Guidelines. Approval for use of the de-identified dataset was obtained from the Human Research Ethics Committee at the WA Department of Health (WADOH HREC\# 2016.24).

Data Availability. WA Health is proprietor of this administrative deidentified dataset. Reasonable requests for data sharing will be forwarded by the authors to WA Health. The manuscript has been reviewed by the custodians of the WA Health Data Linkage Branch registries for appropriateness.

Open Access. This article is licensed under a Creative Commons Attribution-NonCommercial 4.0 International License, which permits any non-commercial use, sharing, adaptation, distribution and reproduction in any medium or format, as long as you give appropriate credit to the original author(s) and the source, provide a link to the Creative Commons licence, and indicate if changes were made. The images or other third party material in this article are included in the article's Creative Commons licence, unless indicated otherwise in a credit line to the material. If material is not included in the article's Creative Commons licence and your intended use is not permitted by statutory regulation or exceeds the permitted use, you will need to obtain permission directly from the 
copyright holder. To view a copy of this licence, visit http://creativecommons.org/licenses/by$\mathrm{nc} / 4.0 /$.

\section{REFERENCES}

1. Ross JJ. Septic arthritis of native joints. Infect Dis Clin North Am. 2017;31:203-18.

2. Goldenberg DL, Reed JI. Bacterial arthritis. N Engl J Med. 1985;312:764-71.

3. Jin T, Mohammad M, Pullerits R, Ali A. Bacteria and host interplay in Staphylococcus aureus septic arthritis and sepsis. Pathogens. 2021;10:158.

4. Fowler VG Jr, Sanders LL, Sexton DJ, et al. Outcome of Staphylococcus aureus bacteremia according to compliance with recommendations of infectious diseases specialists: Experience with 244 patients. Clin Infect Dis. 1998;27:478-86.

5. Lauper N, Davat M, Gjika E, et al. Native septic arthritis is not an immediate surgical emergency. J Infect. 2018;77:47-53.

6. Hassan AS, Rao A, Manadan AM, Block JA. Peripheral bacterial septic arthritis: review of diagnosis and management. J Clin Rheumatol. 2017;23: $435-42$.

7. Ferrand J, El Samad Y, Brunschweiler B, et al. Morbimortality in adult patients with septic arthritis: a three-year hospital-based study. BMC Infect Dis. 2016;16:239.

8. Kaandorp CJ, Krijnen P, Moens HJ, Habbema JD, van Schaardenburg D. The outcome of bacterial arthritis: a prospective community-based study. Arthritis Rheum. 1997;40:884-92.

9. Dadras M, Bohm C, Wallner C, et al. Long-term results of bacterial septic arthritis of the wrist. J Plast Reconstr Aesthet Surg. 2018;71:1138-45.

10. Cohen E, Katz T, Rahamim E, et al. Septic arthritis in children: Updated epidemiologic, microbiologic, clinical and therapeutic correlations. Pediatr Neonatol. 2020;61:325-30.

11. Okubo Y, Nochioka K, Marcia T. Nationwide survey of pediatric septic arthritis in the United States. J Orthop. 2017;14:342-6.

12. Geirsson AJ, Statkevicius S, Vikingsson A. Septic arthritis in iceland 1990-2002: increasing incidence due to iatrogenic infections. Ann Rheum Dis. 2008;67:638-43.
13. McBride S, Mowbray J, Caughey W, et al. Epidemiology, management, and outcomes of large and small native joint septic arthritis in adults. Clin Infect Dis. 2020;70:271-9.

14. Kennedy N, Chambers ST, Nolan I, et al. Native joint septic arthritis: epidemiology, clinical features, and microbiological causes in a new zealand population. J Rheumatol. 2015;42:2392-7.

15. Rutherford AI, Subesinghe S, Bharucha T, Ibrahim F, Kleymann A, Galloway JB. A population study of the reported incidence of native joint septic arthritis in the united kingdom between 1998 and 2013. Rheumatology (Oxford). 2016;55:2176-80.

16. Sreenivas T, Nataraj AR, Kumar A, Menon J. Neonatal septic arthritis in a tertiary care hospital: A descriptive study. Eur J Orthop Surg Traumatol. 2016;26:477-81.

17. Hoswell RL, Johns BP, Loewenthal MR, Dewar DC. Outcomes of paediatric septic arthritis of the hip and knee at 1-20 years in an Australian urban centre. ANZ J Surg. 2019;89:562-6.

18. Goergens ED, McEvoy A, Watson M, Barrett IR. Acute osteomyelitis and septic arthritis in children. J Paediatr Child Health. 2005;41:59-62.

19. Howard-Jones AR, Isaacs D, Gibbons PJ. Twelvemonth outcome following septic arthritis in children. J Pediatr Orthop B. 2013;22:486-90.

20. Nossent J, Raymond W, Keen H, Inderjeeth C, Preen D. Long-term risk of comorbidity after iga vasculitis in childhood: a population-based cohort study. Rheumatol Ther. 2020;7:927-35.

21. Ognjenovic M, Raymond WD, Inderjeeth CA, Keen HI, Preen DB, Nossent JC. The risk and consequences of vertebral fracture in patients with ankylosing spondylitis: a population-based data linkage study. J Rheumatol. 2020;47:1629-36.

22. Quan H, Li B, Couris CM, et al. Updating and validating the Charlson comorbidity index and score for risk adjustment in hospital discharge abstracts using data from 6 countries. Am J Epidemiol. 2011;173:676-82.

23. Knox J, Hume S, Johnson D. Sternoclavicular septic arthritis caused by neisseria elongata subspecies nitroreducens. Pathology. 2015;47:490-2.

24. Nossent JC, Raymond W, Keen H, Preen DB, Inderjeeth CA. Infection rates before and after diagnosis of iga vasculitis in childhood: a population-wide study using non-exposed matched controls. J Rheumatol. 2020;47:424-30. 
25. Riise OR, Handeland KS, Cvancarova M, Wathne $\mathrm{KO}$, Nakstad B, Abrahamsen TG, et al. Incidence and characteristics of arthritis in Norwegian children: a population-based study. Pediatrics. 2008;121:e299-306.

26. Safdieh G, Silberman J, Nguyen J, Doyle SM, Blanco JS, Scher DM, et al. Pediatric septic arthritis and osteomyelitis in the USA: A national KID database analysis. HSS J. 2019;15:159-66.

27. Kim J, Lee MU, Kim TH. Nationwide epidemiologic study for pediatric osteomyelitis and septic arthritis in south korea: A cross-sectional study of national health insurance review and assessment service. Medicine (Baltimore). 2019;98:e15355.

28. Riise OR, Lee A, Cvancarova M, Handeland KS, Wathne KO, Nakstad B, et al. Recent-onset childhood arthritis-association with Streptococcus pyogenes in a population-based study. Rheumatology (Oxford). 2008;47:1006-11.

29. Branson J, Vallejo JG, Flores AR, et al. The contemporary microbiology and rates of concomitant osteomyelitis in acute septic arthritis. Pediatr Infect Dis J. 2017;36:267-73.

30. Perlman MH, Patzakis MJ, Kumar PJ, Holtom P. The incidence of joint involvement with adjacent osteomyelitis in pediatric patients. $\mathrm{J}$ Pediatr Orthop. 2000;20:40-3.

31. Villani MC, Hamilton EC, Klosterman MM, Jo C, Kang LH, Copley LAB. Primary septic arthritis among children 6 to 48 months of age:
Implications for PCR acquisition and empiric antimicrobial selection. J Pediatr Orthop 2021;1;41(3):190-6.

32. Deshpande SS, Taral N, Modi N, Singrakhia M. Changing epidemiology of neonatal septic arthritis. J Orthop Surg (Hong Kong). 2004;12:10-3.

33. Rasmussen L, Bell J, Kumar A, et al. A retrospective review of native septic arthritis in patients: can we diagnose based on laboratory values? Cureus. 2020;12:e8577.

34. Bell J, Rasmussen L, Kumar A, et al. Septic arthritis in immunosuppressed patients: do laboratory values help? J Am Acad Orthop Surg Glob Res Rev 2020;4(3):e20.00007.

35. Spyridakis E, Gerber JS, Schriver E, et al. Clinical features and outcomes of children with culturenegative septic arthritis. J Pediatric Infect Dis Soc. 2019;8:228-34.

36. Williams $\mathrm{N}$, Cooper C, Cundy P.Kingella kingae septic arthritis in children: recognising an elusive pathogen. J Child Orthop. 2014;8:91-5.

37. Jagodzinski NA, Kanwar R, Graham K, Bache CE. Prospective evaluation of a shortened regimen of treatment for acute osteomyelitis and septic arthritis in children. J Pediatr Orthop. 2009;29: 518-25.

38. Paakkonen M. Septic arthritis in children: Diagnosis and treatment. Pediatric Health Med Ther. 2017;8: 65-8. 